Proyecciones

Vol. 23, $\mathrm{N}^{o}$ 2, pp. 81-90, August 2004.

Universidad Católica del Norte

Antofagasta - Chile

\title{
UNIQUENESS OF DETERMINATION OF THE UNKNOWN SOURCE TERM IN SOME MULTIDIMENSIONAL PARABOLIC EQUATIONS
}

\author{
A. ZEGHAL \\ Université Cadi Ayyad, Morocco \\ Received: Abril 2003. Acepted: March 2004.
}

\begin{abstract}
The purpose of this paper is to identify the unknown source term in a multidimensional parabolic equation by means of a one-point interior measurement of the solution at $x_{0} \in \Omega$, i.e. $u\left(x_{0},.\right)$ on $[0, T]$; or a one-point boundary measurement, i.e. $u\left(\bar{x}_{0},.\right)$ on $[0, T]$ with $\bar{x}_{0} \in \partial \Omega$.
\end{abstract}

2000 Mathematics Subject Classification : 35K30, 35K55. 


\section{Introduction}

Let $\Omega$ be an open bounded domain of $\mathbf{R}^{\mathbf{n}}(n \geq 1)$ with $\partial \Omega \in \mathcal{C}^{2+\alpha}$ boundary, $\alpha \in] 0,1[$. Let $T>0$ and let $g=g(x, t)$ be a given function defined on $\partial \Omega \times[0, T]$. We consider the following nonlinear parabolic equation:

$$
\left\{\begin{array}{l}
\partial_{t} u=\Delta u+f(u) \quad \text { in } \bar{\Omega} \times[0, T], \\
u(x, 0)=0 \text { in } \bar{\Omega}, \\
u(x, t)=g(x, t) \quad \text { on } \quad \partial \Omega \times[0, T] .
\end{array}\right.
$$

It is well known that for smooth function $f$, Eq. (1.1) has a unique classical solution, which we denote by $u(f)$, provided the data $g$ is sufficiently regular and satisfies compatibility conditions.

In our case the nonlinear source term $f$ is assumed to be unknown, so that additional information is needed to determine this function.

In this paper, we consider either a one-point interior measurement

$$
u(f)\left(x_{0}, t\right)=\theta(t), \quad t \in[0, T] ;
$$

or a one-point boundary measurement

$$
\partial_{n} u(f)\left(\bar{x}_{0}, t\right)=\bar{\theta}(t), \quad t \in[0, T],
$$

where $x_{0} \in \Omega$ (respectively, $\bar{x}_{0} \in \partial \Omega$ ) and $\partial_{n}$ will denote the derivative with respect to the outward normal to $\partial \Omega$.

More precisely, we are concerned with the unique identifiability of the unknown source term; i.e. with the injectivity of the mapping

$$
f \rightarrow u(f)\left(x_{0}, .\right) \quad \text { or } \quad f \rightarrow \partial_{n} u(f)\left(\bar{x}_{0}, .\right),
$$

which leads to the uniqueness of $f$ in the inverse problems (1.1) - (1.2) or $(1.1)-(1.3)$.

We also study the identifiability (or uniqueness) of the nonlinear term $a$ in the following parabolic equation:

$$
\left\{\begin{array}{l}
\partial_{t} v=a(v) \Delta v \text { in } \bar{\Omega} \times[0, T], \\
v(x, 0)=0 \text { in } \bar{\Omega}, \\
v(x, t)=h(x, t) \text { on } \partial \Omega \times[0, T],
\end{array}\right.
$$

from the observation (1.2) or (1.3). Throughout this paper we denote by $v(a)$ the solution of Eq. (1.4) corresponding to $a$. 
A result in this direction has already been obtained by Choulli [3] for a one-dimensional inverse problem (1.4) - (1.2) (see also, Cannon-DuChateau [1] and DuChateau [2]). In [5], uniqueness results for the determination of the unknown $f$ (respectively, $a$ ) were obtained by Choulli and Zeghal, in the multidimensional case, when the Dirichlet condition is replaced by a Neumann one, from a lateral overdetermination; i.e. $\left.u(f)\right|_{\partial \Omega \times[0, T]}$ (respectively, $\left.\left.v(a)\right|_{\partial \Omega \times[0, T]}\right)$. For an extensive bibliography concerning identifiability problems, the reader is referred to the review article [11] by Nakagiri for a survey of Japanese work up to 1993, and to the survey paper by Isakov [9]. Our results depend heavily on the maximum principles which are contained in the books [8], [12] or [13].

\section{Some properties of $u(f)$ and $v(a)$}

In this section we outline some properties of the solutions of Eqs. (1.1) and (1.4). To this end, we first define

$$
\begin{gathered}
\mathcal{G}=\left\{g \in \mathcal{C}^{2+\alpha, 1+\frac{\alpha}{2}}(\partial \Omega \times[0, T]) ; g(., 0)=\partial_{t} g(., 0)\right\} \\
\widetilde{\mathcal{G}}=\left\{g \in \mathcal{G} ; \partial_{t} g>0, \text { on } \partial \Omega \times(0, T]\right\} \quad \text { and } \quad \mathcal{H}=\left\{h \in \widetilde{\mathcal{G}} ; \partial_{t} h \in \mathcal{G}\right\} .
\end{gathered}
$$

In view of the identifiability problems, we assume throughout that $g \in \widetilde{\mathcal{G}}$ and $h \in \mathcal{H}$.

Let $\mu$ be a positive constant and set

$$
M=\|u(\mu)\|_{\infty} \quad \text { and } \quad N=\|h\|_{\infty} .
$$

For the parabolic equation (1.1) (respectively, Eq. (1.4)), we look for the source term $f$ (respectively, $a$ ) in the set $\mathcal{F}$ (respectively, $\mathcal{A}$ ) consisting of functions $f \in \mathcal{C}^{1}[0, M]$ (respectively, $a \in \mathcal{C}^{1}[0, M]$ ) satisfying $f(0)=0$ and $0 \leq f(s) \leq \mu$ on $[0, M]$ (respectively, $\delta \leq a(s) \leq \gamma$ on $[0, N]$, where $\delta, \gamma$ are positive constants).

It is well known (see, for instance, Ladyzhenskaja et al [10] that if $f \in \mathcal{F}, a \in \mathcal{A}$ and the hypotheses on $g$ and $h$ are satisfied, then Eq. (1.1) (respectively, Eq. (1.4)) has a unique solution $u(f)$ (respectively, $v(a)$ ) in $\mathcal{C}^{2+\alpha, 1+\frac{\alpha}{2}}(\bar{\Omega} \times[0, T])$.

Next, a simple application of the maximum principle leads to the elementary observation. 
Proposition 2.1. Let $f \in \mathcal{F}$ and $a \in \mathcal{A}$. Then $\mathcal{R}(u(f)) \subset[0, M]$ and $\mathcal{R}(v(a))=[0, N]$, where $\mathcal{R}$ stands for a range of a function.

Proof. Let $f \in \mathcal{F}$. Then the maximum principle applied successively to $-u(f), u(f)-u(\mu)$, together with (2.1) leads to the following

$$
0 \leq u(f)(x, t) \leq u(\mu)(x, t) \leq M, \text { on } \bar{\Omega} \times[0, T] .
$$

Thus the range of $u(f)$ is contained in the domain of $f$. In a similar way, we obtain the second assertion.

We will use the following Lemma.

Lemma 2.1. Let $f \in \mathcal{F}$ and $g \in \widetilde{\mathcal{G}}$. Then, for all $s_{1}>0$, there exists $s_{0}>0$ and $T_{0}, T_{1}$ with $0<T_{0}<T_{1} \leq T$ such that

$$
s_{0} \leq u(f)(x, t) \leq s_{1} \quad \text { on } \quad \bar{\Omega} \times\left[T_{0}, T_{1}\right] .
$$

Proof. Let $s_{1}>0$. It follows from the regularity of $u(\mu), u(\mu)(x, 0)=0$, and $(2.2)$ that $u(f) \leq k t$ on $\bar{\Omega} \times[0, T]\left(k=\left\|\partial_{t} u(\mu)\right\|_{\infty}\right)$. Thus, there exists $T_{1}, 0<T_{1} \leq T$ such that $u(f)(x, t) \leq s_{1}$ on $\bar{\Omega} \times\left[0, T_{1}\right]$.

Now, let $\psi=\psi(x, t) \in \mathcal{C}^{2+\alpha, 1+\frac{\alpha}{2}}\left(\bar{\Omega} \times\left[0, T_{1}\right]\right)$ be the solution of the following parabolic equation:

$$
\left\{\begin{array}{l}
\partial_{t} \psi-\Delta \psi=0 \text { in } \bar{\Omega} \times\left[0, T_{1}\right] \\
\psi(x, 0)=0 \text { in } \bar{\Omega}, \\
\psi(x, t)=g(x, t) \quad \text { on } \quad \partial \Omega \times\left[0, T_{1}\right]
\end{array}\right.
$$

and let $\psi_{h}$ be the function defined by $\psi_{h}(x, t)=\psi(x, t+h)-\psi(x, t)$ on $\bar{\Omega} \times\left[0, T_{1}-h\right]$ for $0<h<T_{1}$. Then $\psi_{h}$ is the solution of the following equation:

$$
\left\{\begin{array}{l}
\partial_{t} \psi_{h}-\Delta \psi_{h}=0 \text { in } \bar{\Omega} \times\left[0, T_{1}-h\right] \\
\psi_{h}(x, 0)=\psi(x, h) \geq 0 \quad \text { in } \bar{\Omega} \\
\psi_{h}(x, t)=g(x, t+h)-g(x, t) \geq 0 \quad \text { on } \quad \partial \Omega \times\left[0, T_{1}-h\right]
\end{array}\right.
$$

By the maximum principle applied to $-\psi_{h}$, we obtain $\psi_{h} \geq 0$ on $\bar{\Omega} \times\left[0, T_{1}-\right.$ $h]$. Passing to the limit we deduce that $\partial_{t} \psi \geq 0$ on $\bar{\Omega} \times\left[0, T_{1}\right]$ and therefore

$$
\psi(x, t) \geq \min \{\psi(y, \tau) ; y \in \bar{\Omega}\}>0, \quad 0<\tau \leq t \leq T_{1} \text { and } x \in \bar{\Omega},
$$

(the second inequality follows from an application of the maximum principle to $\psi$ and the hypotheses on $g$ ). Since, $\min \{\psi(y, \tau) ; y \in \bar{\Omega}\} \rightarrow 0$ as $\tau \rightarrow 0$, 
one sees that there exists $\left.\left.T_{0} \in\right] 0, T_{1}\right]$ and $s_{0}>0$ such that $s_{0} \leq \psi(x, t) \leq s_{1}$ on $\bar{\Omega} \times\left[T_{0}, T_{1}\right]$.

Finally, using again the maximum principle to the function $\psi-u(f)$, we deduce that $u(f) \geq \psi$ on $\bar{\Omega} \times\left[0, T_{1}\right]$, which achieves the proof.

Lemma 2.2. Let $a \in \mathcal{A}$ and $h \in \mathcal{H}$. Then the following hold.

(i) $\Delta v(a)>0$ on $\bar{\Omega} \times(0, T]$.

(ii) For all $s_{1}>0$, there exists $s_{0}>0$ and $T_{0}, T_{1}$ with $0<T_{0}<T_{1} \leq T$ such that

$$
s_{0} \leq v(a)(x, t) \leq s_{1} \quad \text { on } \quad \bar{\Omega} \times\left[T_{0}, T_{1}\right]
$$

Proof. (i) Let $w=e^{-\lambda t} \partial_{t} v(a)$, where $\lambda \in \mathbf{R}$ is to be selected in the sequel. A straightforward calculations show that $w$ is a solution of the following equation:

$$
\left\{\begin{array}{l}
a(v) \Delta w+(c(x, t)-\lambda) w-\partial_{t} w=0 \quad \text { in } \bar{\Omega} \times[0, T] \\
w(x, 0)=0 \text { in } \bar{\Omega}, \\
w(x, t)=e^{-\lambda t} \partial_{t} h(x, t) \quad \text { on } \quad \partial \Omega \times[0, T]
\end{array}\right.
$$

where $c=c(x, t)=a^{\prime}(v(a)(x, t)) \Delta v(a)(x, t)$. Since, $c$ is bounded, we can choose $\lambda$ so large that $c-\lambda \leq 0$. Then, by a maximum principle applied to $-w$, we have $w>0$ on $\bar{\Omega} \times(0, T]$ (because $\partial_{t} h(x, t)>0$ on $\partial \Omega \times(0, T]$ ), and consequently

$$
\Delta v(a)=\frac{w}{a(v)} e^{\lambda t}>0 \quad \text { on } \quad \bar{\Omega} \times(0, T] .
$$

(ii) By the maximum principle applied successively to $v(\delta)-v(a)$ and $v(a)-$ $v(\gamma)$ and the fact that $\Delta v(\delta)$ and $\Delta v(\gamma)$ are positive, we deduce

$$
v(\delta)(x, t) \leq v(a)(x, t) \leq v(\gamma)(x, t) \leq k t \quad \text { on } \quad \bar{\Omega} \times[0, T]
$$

where $k$ is a positive constant. The last inequality of (2.5) follows from the regularity of $v(\gamma)$ and the fact that $v(\gamma)(x, 0)=0$.

Now, the rest of the proof is similar to that of Lemma 2.1, it suffices to replace the function $\psi$ by $v(\delta)$. 


\section{The main results}

Before stating our main results, let us make some notations. Let $\mathcal{F}_{a}$ (respectively, $\mathcal{A}_{a}$ ) be the set of real analytic functions of $\mathcal{F}$ (respectively, $\mathcal{A})$.

In the case of a one-point interior measurement, we have the following result.

Theorem 3.1. (i) Let $g \in \widetilde{\mathcal{G}}$ and $f_{1}, f_{2} \in \mathcal{F}_{a}$. If $u\left(f_{1}\right)\left(x_{0},.\right)=u\left(f_{2}\right)\left(x_{0},.\right)$ on $[0, T]$, then $f_{1}=f_{2}$.

(ii) Let $h \in \mathcal{H}$ and let $a_{1}, a_{2} \in \mathcal{A}_{a}$. If $v\left(a_{1}\right)\left(x_{0},.\right)=v\left(a_{2}\right)\left(x_{0},.\right)$ on $[0, T]$, then $a_{1}=a_{2}$.

From this result we immediately obtain uniqueness of the solution of the inverse problem (1.2).

Corollary 3.1. Under the hypotheses $g \in \widetilde{\mathcal{G}}$ and $h \in \widetilde{\mathcal{H}}$, the inverse problem (1.2)associated to Eq. (1.1) (respectively, Eq. (1.4)), can possess at most one solution in $\mathcal{F}_{a}$ (respectively, in $A_{a}$ ).

Proof of Theorem 3.1. (i) Let $g \in \widetilde{\mathcal{G}}$ and let $f_{1}, f_{2} \in \mathcal{F}_{a}$. Assume that

$$
u\left(f_{1}\right)\left(x_{0}, .\right)=u\left(f_{2}\right)\left(x_{0}, .\right) \quad \text { on } \quad[0, T] .
$$

We proceed by contradiction to prove that $f_{1}=f_{2}$. To do so, assume this is not true, i.e. $f_{1} \neq f_{2}$ on $[0, M]$. Since the zeros of an analytic function are isolated, there exists $s_{1}>0,0<s_{1} \leq M$, such that $f_{1}-f_{2}$ is not identically equal to zero on $\left(0, s_{1}\right]$. We assume, without loss of generality, that $f_{1}(s)>f_{2}(s)$ for all $s \in\left(0, s_{1}\right]$.

Let $w=e^{-\lambda t}\left(u\left(f_{1}\right)-u\left(f_{2}\right)\right)$, where $\lambda \in \mathbf{R}$. By a straightforward computation, we obtain the equation

$$
\left\{\begin{array}{l}
\Delta w-\partial_{t} w+(c(x, t)-\lambda) w=-F(x, t) \text { in } \bar{\Omega} \times[0, T] \\
w(x, 0)=0 \text { in } \bar{\Omega} \\
w(x, t)=0 \text { on } \partial \Omega \times[0, T],
\end{array}\right.
$$

where

$$
F=F(x, t)=e^{-\lambda t}\left\{f_{1}\left(u\left(f_{2}\right)(x, t)\right)-f_{2}\left(u\left(f_{2}\right)(x, t)\right)\right\},
$$


and

$$
c=c(x, t)=\int_{0}^{1} f_{1}^{\prime}\left(s u\left(f_{1}\right)(x, t)+(1-s) u\left(f_{2}\right)(x, t)\right) d s
$$

is a bounded function. From Lemma 2.1, there exists $s_{0}>0$ and $T_{0}, T_{1}$ with $0<T_{0}<T_{1} \leq T$ such that $s_{0} \leq u\left(f_{2}\right)(x, t) \leq s_{1}$ on $\bar{\Omega} \times\left[T_{0}, T_{1}\right]$. So that

$$
F>0 \text { on } \bar{\Omega} \times\left[T_{0}, T_{1}\right] .
$$

Now, let $\lambda$ be so large that $c-\lambda \leq 0$. Consequently, $w \geq 0$ on $\bar{\Omega} \times\left[0, T_{1}\right]$ (use the maximum principle to $-w$ ). We deduce that $\min _{\left[T_{0}, T_{1}\right]} w=0$ is attained on $\left\{x_{0}\right\} \times\left[T_{0}, T_{1}\right]$. Since $x_{0} \in \Omega=\operatorname{Int}(\Omega)$, it follows from the minimum principle that $w=0$ on $\bar{\Omega} \times\left[T_{0}, T_{1}\right]$, which is in contradiction with (3.1)-(3.2).

(ii) Let $h \in \mathcal{H}$ and let $a_{1}, a_{2} \in \mathcal{A}_{a}$. Assume that $v\left(a_{1}\right)\left(x_{0},.\right)=$ $v\left(a_{2}\right)\left(x_{0},.\right)$ on $[0, T]$ and $a_{1} \neq a_{2}$. Proceeding as in the proof of the first item; we assume, without loss of generality, that $a_{1}(s)>a_{2}(s)$ on $\left(0, s_{1}\right]$. Thus, there exists $s_{0}>0$ and $T_{0}, T_{1}$ with $0<T_{0}<T_{1} \leq T$ such that $s_{0} \leq v\left(a_{2}\right)(x, t) \leq s_{1}$ on $\bar{\Omega} \times\left[T_{0}, T_{1}\right]$.

We obtain for $w=e^{-\lambda t}\left(v\left(a_{1}\right)-v\left(a_{2}\right)\right)$ the following equation

(3.3) $\left\{\begin{array}{l}a_{1}\left(v\left(a_{1}\right)\right) \Delta w-\partial_{t} w+(c(x, t)-\lambda) w=-F(x, t) \text { in } \bar{\Omega} \times[0, T] \\ w(x, 0)=0 \text { in } \bar{\Omega} \\ w(x, t)=0 \text { on } \partial \Omega \times[0, T]\end{array}\right.$

where

$$
\begin{aligned}
& F=F(x, t)=e^{-\lambda t}\left\{a_{1}\left(v\left(a_{2}\right)(x, t)\right)-a_{2}\left(v\left(a_{2}\right)(x, t)\right)\right\} \Delta v_{2}\left(a_{2}\right)(x, t), \\
& c=c(x, t)=\Delta v\left(a_{2}\right)(x, t) \int_{0}^{1} a_{1}^{\prime}\left(s v\left(a_{1}\right)(x, t)+(1-s) v\left(a_{2}\right)(x, t)\right) d s,
\end{aligned}
$$

and $\lambda \geq\|c\|_{\infty}$. Using Lemma 2.2(i), we deduce that $F>0$ on $\bar{\Omega} \times\left[T_{0}, T_{1}\right]$. Now, the rest of the proof is similar to the previous one.

Remark 3.1 Let $\mathcal{A}_{c} \subset \mathcal{C}^{1}[0, N]$ be the set of all function a such that $a^{-1}\{0\}$ has no accumulation point. Then we have the following corollary.

Corollary 3.2. Let $h \in \mathcal{H}$ and let $a_{1}, a_{2} \in \mathcal{A}$. Assume that $a_{1}-a_{2} \in \mathcal{A}_{c}$. If $v\left(a_{1}\right)\left(x_{0},.\right)=v\left(a_{2}\right)\left(x_{0},.\right)$ on $[0, T]$, then

$$
\max _{\bar{\Omega} \times[0, T]} v\left(a_{1}\right)=\max _{\bar{\Omega} \times[0, T]} v\left(a_{2}\right)=m \quad \text { and } \quad a_{1}=a_{2} \text { on }[0, m] .
$$


Note that the condition $a_{1}-a_{2} \in \mathcal{A}_{c}$, in the previous corollary, occurs frequently in the inverse problems where the unknown is the nonlinear term appearing in the equation (see, for instance, [7], [5], [2] and [4]).

In the case of a one-point boundary measurement, we have the following result.

Theorem 3.2. (i) Let $g \in \widetilde{\mathcal{G}}$ and let $f_{1}, f_{2} \in \mathcal{F}_{a}$. If $\partial_{n} u\left(f_{1}\right)\left(\bar{x}_{0},.\right)=$ $\partial_{n} u\left(f_{2}\right)\left(\bar{x}_{0},.\right)$ on $[0, T]$, then $f_{1}=f_{2}$.

(ii) Let $h \in \mathcal{H}$ and let $a_{1}, a_{2} \in \mathcal{A}_{a}$. If $\partial_{n} v\left(a_{1}\right)\left(\bar{x}_{0},.\right)=\partial_{n} v\left(a_{2}\right)\left(\bar{x}_{0},.\right)$ on $[0, T]$, then $a_{1}=a_{2}$.

Proof. Let $g \in \widetilde{\mathcal{G}}$ and let $f_{1}, f_{2} \in \mathcal{F}_{a}$. Assume that

$$
\partial_{n} u\left(f_{1}\right)\left(\bar{x}_{0}, .\right)=\partial_{n} u\left(f_{2}\right)\left(\bar{x}_{0}, .\right) \quad \text { on } \quad[0, T] .
$$

We argue as in the proof of Theorem 3.1. Suppose that $f_{1} \neq f_{2}$. Since the minimum of the solution $w$ of Eq. (3.1) is attained on $\left\{\bar{x}_{0}\right\} \times[0, T] \subset$ $\partial \Omega \times[0, T]$ and $w$ is not constant on $\bar{\Omega} \times\left[T_{0}, T_{1}\right]\left(\right.$ since $F>0$ on $\left.\bar{\Omega} \times\left[T_{0}, T_{1}\right]\right)$, it follows from the minimum principle that

$$
\partial_{n} w<0 \quad \text { on } \quad\left\{\bar{x}_{0}\right\} \times\left[T_{0}, T_{1}\right],
$$

which is in contradiction with (3.4).

The proof of the second item is similar.

The following result is immediate from the theorem above.

Corollary 3.3. Under the hypotheses of Corollary 3.1, the inverse problem (1.3) associated to Eq. (1.1) (respectively, Eq. (1.4)), can possess at most one solution in $\mathcal{F}_{a}$ (respectively, in $\mathcal{A}_{a}$ ).

Acknowledgement. The author would like to thank Professor M. Choulli for many useful discussions. 


\section{References}

[1] J. R. Cannon and P. DuChateau, An inverse problem for a nonlinear diffusion equation, SIAM J. Appl. Math. 39, pp. 272-289, (1980).

[2] J. R. Cannon and P. DuChateau, Structural identification of an unknown source term in a heat equation, Inverse problems 14, pp. 535551, (1998).

[3] M. Choulli, Identifiability of an unknown coefficient in a nonlinear diffusion equation, Quart. Appl. Math. 47, pp. 9-16, (1989).

[4] M. Choulli, On the determination of an unknown boundary function in a parabolic equation, Inverse problems 15, pp. 659-667, (1999).

[5] M. Choulli and A. Zeghal, Un résultat d'unicité pour un problème inverse parabolique semi-linéaire, C. R. Acad. Sci. Paris Sér. I 315, pp. 1051-1053, (1992).

[6] P. DuChateau, Monotonicity and uniqueness results in identifying an unknown coefficient in a nonlinear diffusion equation, Siam J. Appl. Math. 41, pp. 310-323, (1981).

[7] P. DuChateau and W. Rundell, Unicity in an inverse problem for an unknown reaction term in a reaction-diffusion equation, J. Diff. Equations 59, pp. 155-164, (1985).

[8] A. Friedman, Partial Differential Equations of Parabolic Type , Prentice-Hall, Englewood Cliffs, NJ, (1964).

[9] V. Isakov, Uniqueness and stability in multi-dimensional inverse problems, Inverse Problems 9, pp. 579-621, (1993).

[10] O. A. Ladyzhenskaja, V. A. Solonnikov and N. N. Ural'ceva, Linear and Quasi-linear Equations of Parabolic Type, Providence, RI, (1968).

[11] S. Nakagiri, Review of Japanese work of the last ten years on identifiability in distributed parameter systems, Inverse Problems 9, pp. 143-191, (1993). 
[12] M. H. Protter and H. F. Weinberger, Maximum Principles in Differential Equations, Springer-Verlag, New York, (1984).

[13] M. Renardy and R. C. Rogers An Introduction to Partial Differential Equations, Springer-Verlag, New York, (1993).

\author{
A. ZEGHAL \\ Department of Applied Mathematics \\ Faculté des Sciences et Techniques \\ Université Cadi Ayyad \\ B. P. 523 \\ Beni - Mellal \\ Morocco \\ email : zeghal@yahoo.com
}

